\title{
Prevalência dos casos de sífilis em gestantes no Brasil: Análise de uma década
}

Prevalence of syphilis cases in pregnant women in Brazil: A decade analysis

Prevalencia de casos de sífilis en mujeres embarazadas en Brasil: Análisis de una década

Airton César Leite

ORCID: https://orcid.org/0000-0001-7184-8488

Centro Universitário Santo Agostinho, Brasil E-mail: ainton.cesar2014@gmail.com

Mariana Pereira Barbosa Silva

ORCID: https://orcid.org/0000-0003-0852-8099

Universidade Estadual do Piauí, Brasil

E-mail: marianapbsilvaa@gmail.com

Danielle Sousa Almeida

ORCID: https://orcid.org/0000-0002-0537-8972

Centro Universitário Santo Agostinho, Brasil

E-mail: daniellyalmeida99@gmail.com

Juliana Torres Avelino

ORCID: https://orcid.org/0000-0002-8732-1856

Centro Universitário Santo Agostinho, Brasil

E-mail: juliana_avelinno@hotmail.com

Flávia Nunes Barbosa

ORCID: https://orcid.org/0000-0002-8101-2032 Universidade Federal do Piauí, Brasil E-mail: flavianunes-cat@ hotmail.com

Geovana Maria Rodrigues de Sousa

ORCID: https://orcid.org/0000-0001-6398-8560

Centro Universitário Santo Agostinho, Brasil

E-mail: geovanamaria08@hotmail.com

Samuel Lopes dos Santos

ORCID: https://orcid.org/0000-0003-3375-9171 Universidade Federal do Piauí, Brasil

E-mail: samuellopes121314@gmail.com

Laiana Dias Prudêncio

ORCID: https://orcid.org/0000-0002-0016-3868

Centro Universitário Maurício de Nassau, Brasil

E-mail: laianadias568@gmail.com

Raimunda da Silva Sousa Neta

ORCID: https://orcid.org/0000-0001-5927-0046

Centro Universitário Santo Agostinho, Brasil

E-mail: raynettasousa@gmail.com

Mariane Gomes Duarte de Sousa

ORCID: https://orcid.org/0000-0002-3218-9704

Centro Universitário Santo Agostinho, Brasil

E-mail: duartemariane7@gmail.com

Weberton Dorásio Sobrinho

ORCID: https://orcid.org/0000-0003-0619-7214

Universidade de Rio Verde, Brasil

E-mail: dorasioweberton@gmail.com

Johnny Lima Brandão

ORCID: https://orcid.org/0000-0003-4039-8079

Universidade Federal do Estado do Rio de Janeiro, Brasil

E-mail: johnny.brandao@edu.unirio.br

Andréia Santos de Carvalho Pinheiro

ORCID: https://orcid.org/0000-0002-6506-6065

Centro Universitário Santo Agostinho, Brasil

E-mail: andreiascarvalho2018@gmail.com

Alaine dos Santos Silva Martins

ORCID: https://orcid.org/0000-0002-1898-4920

Universidade Estadual de Ciências da Saúde de Alagoas, Brasil

E-mail: alaine.martins22@gmail.com

Vanessa Lorrana Correia de Sousa

ORCID: https://orcid.org/0000-0001-8159-6763

Associação de Ensino Superior do Piauí, Brasil

E-mail: enfervanessalorrana@gmail.com 


\title{
Nágila Silva Alves \\ ORCID: https://orcid.org/0000-0002-1618-8111 Centro Universitário Santo Agostinho, Brasil Email: nglarraial@gmail.com \\ Mariana Tenório Costa \\ ORCID: https://orcid.org/0000-0001-8116-4864 Centro Universitário Tiradentes, Brasil E-mail: mtc0809@hotmail.com \\ Sijomara Maria Costa Freitas ORCID: https://orcid.org/0000-0003-3443-7334 Centro Universitário Uninassau Redenção, Brasil E-mail: sijomara.costa@icloud.com \\ Erica Williams de Moreira Lima \\ ORCID: https://orcid.org/0000-0003-3957-5699 \\ Centro Universitário Uninovafapi, Brasil \\ E-mail: williams-erica1992@outlook.com \\ Layanne Cavalcante de Moura \\ ORCID: https://orcid.org/0000-0003-2781-1076 Centro Universitário UNIFACID, Brasil \\ E-mail: layannecavalcante@hotmail.com \\ Joelma Maria dos Santos da Silva Apolinário ORCID: https://orcid.org/0000-0001-9521-9432 Faculdade Maurício de Nassau, Brasil E-mail: jo.silva00@hotmail.com \\ Moacir Andrade Ribeiro Filho \\ ORCID: https://orcid.org/0000-0003-1991-469X Universidade Regional do Cariri, Brasil E-mail: moacirarf@outlook.com Marianna Leite Barroso ORCID: https://orcid.org/0000-0002-2147-7014 Faculdade São Francisco, Brasil E-mail: mariannaleite_@hotmail.com
}

\begin{abstract}
Resumo
Analisar a prevalência de casos de sífilis em gestantes no Brasil: análise de uma década. Trata-se de um estudo epidemiológico, observacional, descritivo, do tipo documental com abordagem quanti-qualitativa, realizado através do levantamento de dados secundários do SINAN, disponível na plataforma eletrônica do Departamento de Informática do Sistema Único de Saúde (DATASUS) (Brasil, 2021). A sífilis gestacional vem ocorrendo com frequência destacando-se alguns fatores que contribuem, estes são: classe média baixa, baixo índice de escolaridade, déficit no acompanhamento pré-natal, sendo este um dos mais importantes para o desfecho de intervir no resultado, adquirida no protocolo da gestante, garantido desta forma uma assistência qualificada. A sífilis é uma patologia multifacetada, com sérias implicações para a mulher grávida e seu concepto. Quando adquirida durante a gravidez, pode levar a abortamento espontâneo, morte fetal e neonatal, prematuridade e danos à saúde do recém-nascido com repercussões psicológicas e sociais. Evidencia-se que, a educação em saúde é importante no desfecho de uma gestação, sendo o profissional enfermeiro, capaz de auxiliar a gestante a viver de forma mais saudável e com todos os cuidados necessários nesse período da sua vida. Portanto, é necessário realizar ações de promoção à saúde na gestação, dando enfoque ao autocuidado e prevenção, pois reduzem os gastos com assistência de maior complexidade e também diminuem as complicações obstétricas e neonatais que podem ser causadas pela sífilis.
\end{abstract}

Palavras-chave: Cuidados de enfermagem; Gestantes; Sífilis.

\begin{abstract}
To analyze the prevalence of syphilis cases in pregnant women in Brazil: a decade analysis. This is an epidemiological, observational, descriptive, documentary-type study with a quanti-qualitative approach, carried out through the survey of secondary data from SINAN, available on the electronic platform of the Information Technology Department of the Unified Health System (DATASUS) (Brazil, 2021). Gestational syphilis has been occurring frequently, highlighting some contributing factors, which are: lower middle class, low level of education, deficit in prenatal care, which is one of the most important for the outcome of intervening in the result, acquired in the protocol of the pregnant woman, thus guaranteeing a qualified assistance. Syphilis is a multifaceted pathology, with serious implications for the pregnant woman and her fetus. When acquired during pregnancy, it can lead to spontaneous abortion, fetal and neonatal death, prematurity and damage to the health of the newborn, with psychological and social repercussions. It is evident that health education is important in the outcome of a pregnancy, with the professional nurse being able to help the pregnant woman to live healthier and with all the necessary care during this period of her life. Therefore, it is necessary to carry out health promotion actions during pregnancy, focusing on self-care and prevention, as they reduce expenditure on more complex care and also reduce obstetric and neonatal complications that can be caused by syphilis.
\end{abstract}


Keywords: Nursing care; Pregnant women; Syphilis.

\section{Resumen}

Analizar la prevalencia de casos de sífilis en gestantes en Brasil: análisis de una década. Se trata de un estudio epidemiológico, observacional, descriptivo, de tipo documental con enfoque cuanti-cualitativo, realizado a través de la encuesta de datos secundarios del SINAN, disponible en la plataforma electrónica del Departamento de Tecnología de la Información del Sistema Único de Salud (DATASUS) (Brasil, 2021). La sífilis gestacional ha venido ocurriendo con frecuencia, destacando algunos factores contribuyentes, los cuales son: clase media baja, bajo nivel educativo, déficit en la atención prenatal, que es uno de los más importantes para el desenlace de intervenir en el resultado, adquirido en el protocolo de la mujer embarazada, garantizando así una asistencia calificada. La sífilis es una patología multifacética, con graves implicaciones para la embarazada y su feto. Cuando se adquiere durante el embarazo, puede provocar abortos espontáneos, muerte fetal y neonatal, prematurez y daños a la salud del recién nacido, con repercusiones psicológicas y sociales. Es evidente que la educación para la salud es importante en el desenlace de un embarazo, pudiendo la enfermera profesional ayudar a la gestante a vivir más saludable y con todos los cuidados necesarios durante este período de su vida. Por tanto, es necesario realizar acciones de promoción de la salud durante el embarazo, con foco en el autocuidado y la prevención, ya que reducen el gasto en cuidados más complejos y también reducen las complicaciones obstétricas y neonatales que puede ocasionar la sífilis.

Palabras clave: Cuidado de enfermera; Mujeres embarazadas; Sífilis.

\section{Introdução}

A gestação é uma fase importante na vida da mulher, é um momento de mudanças físicas acompanhadas de alterações emocionais. É fundamental que o pré-natal tenha início precoce, medidas preventivas podem ter alcance maior e o tratamento de certas intercorrências pode ser realizado antes de qualquer comprometimento tanto da gestante como do feto. As infecções sexualmente transmissíveis (IST’s) são consideradas um problema de saúde pública e estão entre as patologias transmissíveis mais comuns, afetando a saúde e a vida das pessoas em todo o mundo. As IST's têm um impacto direto sobre a saúde reprodutiva e infantil, porquanto acarretam infertilidade e complicações na gravidez e no parto, além de causar morte fetal e agravos à saúde da criança (Nesi, Graf, \& Moraes, 2020).

As infecções maternas decorrentes de micro-organismos que são passíveis de transmissão ao feto constituem uma das principais possibilidades para aumento e manutenção dos altos índices de morbimortalidade perinatal. As maiores taxas de transmissão dessas infecções concentram-se na sífilis, uma doença sistêmica definida como problema de saúde pública em virtude da alta frequência de condução e graves desfechos para a gestação e o feto, como abortamentos, perdas fetais tardias e óbitos neonatais. Assim, os impactos pessoais, familiares e sociais decorrentes da amplitude dessa doença exigem uma assistência multidimensional ao binômio mãe-filho que contemple todos os aspectos inerentes ao processo de cuidar. Nessa perspectiva, a possibilidade da construção de uma linha de cuidados abrange o trabalho em rede entre diferentes profissionais, em diversos serviços de saúde e setores sociais (Araújo et al., 2019).

De acordo com Souza et al. (2018), a sífilis é uma Infecção Sexualmente Transmissível (IST) causada pelo Treponema pallidum (T. pallidum) com formato de espiroquetas (delgadas, gram negativas). É um agravo sistêmico, de evolução lenta e crônica. O processo de transmissão ocorre a partir do contato direto com as lesões que pode ocorrer por meio de transfusão sanguínea, contato sexual, transmissão vertical (gestantes e parturientes) e através de acidentes com material biológico contaminado.

Segundo Gomes, Oliveira, e Brandão (2016), a sífilis gestacional é considerada quando toda gestante com evidência clínica de sífilis e/ou com sorologia não treponêmica reagente, com qualquer titulagem, mesmo na ausência de resultado de teste treponêmico, realizada no pré-natal ou no momento do parto ou curetagem.

No Brasil, a estimativa é que a média de prevalência de sífilis em gestantes varia entre 1,4\% e 2,8\%, com uma taxa de transmissão vertical de $25 \%$ e cerca de 900 mil casos por ano. Nota-se ainda que, a sífilis no período gestacional teve um 
aumento de quase oito mil para mais de 28 mil casos, e que o número de bebês infectados cresceu de 5,7 mil para mais de 16 mil (Oliveira, Nunes, \& Andrade, 2017).

A sífilis congênita é uma infecção do feto em decorrência da passagem do treponema pela placenta, sendo mais grave quanto acomete a gestante no primeiro trimestre de gestação, caracterizando as principais causas de aborto em todo o mundo segundo a Organização Mundial da Saúde.

Destacando-se a sífilis, o que torna-se imprescindível a implantação de medidas visando o controle dos casos e, posteriormente, a erradicação da doença no país. A sífilis apresenta-se em distribuição mundial, constituindo assim no mundo e no Brasil um importante problema de saúde pública. Dessa forma, estima-se que grande parte das gestações com sífilis não tratada evoluam para perda fetal e que mais de $50 \%$ dos recém-nascidos de mães com sífilis não tratada ou tratada de forma inadequada, não apresentem sinais evidentes da doença logo ao nascimento, retardando o seu diagnóstico e, produzindo consequentemente, complicações para a criança no futuro (Gomes, Oliveira, \& Brandão, 2016).

Algumas evidências apontam que o aumento dos casos de sífilis está diretamente ligado a questões sociais e econômicas. Os casos de sífilis congênita, por exemplo, estão associados, na maioria das vezes, com à menor escolaridade materna, cor da pele preta, maior proporção de fatores de risco para prematuridade, início mais tardio do pré-natal ou não realização do pré-natal, menor número de consultas e menor realização de exames sorológicos. Diante disso, a não realização do tratamento ou o tratamento inadequado dessa infecção treponêmica, faz que a mesma evolua para formas mais graves. Essa evolução acaba comprometendo o sistema nervoso, o aparelho cardiovascular, o aparelho respiratório, o aparelho gastrointestinal e, na gestação, pode acarretar em prematuridade, abortamento espontâneo e óbito fetal, tendo em vista que cerca de $40 \%$ das gestações que cursam com sífilis resultam em morte do concepto (Solino et al., 2020).

A sífilis em gestante se tornou uma doença de notificação compulsória pelo seu agravo e em busca de controlar a sua transmissão na população, instituindo assim políticas de saúde pública. A notificação foi instituída pela portaria n³3 de 14 de junho de 2005. Na primeira consulta de pré-natal a gestante deve fazer o exame para a detecção da infecção da sífilis e repetir no $3^{\circ}$ trimestre de gestação e horas antes do parto (independentemente dos resultados anteriores), e em caso de abortamento. O profissional da atenção primária de saúde tem a responsabilidade de fornecer um plano assistencial às gestantes diagnosticada com sífilis, visando quebrar a cadeia de transmissão comunitária e do binômio mãe-filho. Pois com uma assistência inadequada e com um tratamento tardio, as consequências resultam em mortes fetais e neonatais precoces e alta probabilidade de transmissão vertical (mãe-filho), principalmente na fase primária e secundária (Silva Júnior, Lima, \& Aramaio, 2021).

Dessa forma, o acompanhamento é imprescindível à gestante com sífilis. Nesse momento, o enfermeiro que atende estas mulheres interroga sobre seu estado, avalia a efetividade e adesão ao tratamento, como também do parceiro. Assim, a captação e as orientações às gestantes e parceiros por parte do enfermeiro possui contribuição decisiva para ações eficientes no combate à sífilis, de maneira a ser implementada relação harmoniosa e ética sustentada no compromisso seguro da usuária com resolubilidade de seus problemas (Nunes et al., 2017).

O enfermeiro é importante no controle da sífilis gestacional informando sobre o tratamento de forma correta; uso de preservativos nas relações sexuais; promover educação em saúde; prática de exames; e captação dos parceiros. Torna-se necessário melhorar a qualidade do acompanhamento pré-natal, a partir da capacitação dos profissionais envolvidos, enfatizando a importância da notificação dos casos de sífilis em gestantes visando ao monitoramento do problema e avaliação das ações propostas (Nunes et al., 2017).

O tratamento e a detecção da sífilis na gestação são representativas de grande importância para a saúde pública, sendo essencial para a prevenção e o controle desta patologia. Os profissionais enfermeiros possuem papel fundamental quanto à aplicação de estratégias que auxiliem na prevenção e no diagnóstico da sífilis congênita, assim como, reduzir os riscos de 
mortalidade e morbidade materna e fetal. Desta forma, os profissionais enfermeiros, enquanto indivíduos assistenciais para o cuidado no pré-natal, desenvolvem papel fundamental na implementação e promoção de medidas que precisam ter sua aplicabilidade de forma generalizada e sistêmica, havendo o enfrentamento desse problema, assim como a aplicação da terapêutica recomendada (Leite, Oliveira, Leão, Lopes, \& França, 2016).

Com o propósito de promover um cuidado adequado à mulher durante seu período gestacional, a consulta de enfermagem surge como possibilidade de originar mudanças, sendo o enfermeiro o profissional capaz de intervir diretamente na saúde dessa mulher agregando à consulta a aplicação da Sistematização da Assistência de Enfermagem (SAE), a fim de possibilitar um redesenho do cuidado de enfermagem que visa a detecção precoce da sífilis durante o período gestacional (Felicío et al., 2019).

Diante da temática em discussão, surge como questão norteadora do estudo: "Qual a prevalência de casos de sífilis em gestantes no Brasil: análise de uma década"? Assim, o objetivo do presente estudo é analisar a prevalência de casos de sífilis em gestantes no Brasil: análise de uma década.

A realização do presente estudo relacionado à temática, é essencial para a construção coletiva do conhecimento, pois, o estudo possibilita a sociedade acadêmica e cientifica a ampliar o conhecimento sobre a prevalência de casos de sífilis em gestantes no Brasil: análise de uma década. Desse modo, o estudo trará contribuições pertinentes, na medida em que acrescentará as evidências científicas discussões relevantes a respeito dessa temática tão importante.

\section{Metodologia}

Trata-se de um estudo epidemiológico, observacional, descritivo, do tipo documental com abordagem quantiqualitativa, realizado através do levantamento de dados secundários do SINAN, disponível na plataforma eletrônica do Departamento de Informática do Sistema Único de Saúde (DataSUS) (Brasil, 2021), referentes aos casos de sífilis em gestantes no Brasil: análise de uma década, no período de 2010 a 2020. O presente estudo encontra suporte metodológico nas bases de dados em pesquisas científicas debatidas por Pereira et al. (2018).

Para a coleta de dados, realizada entre os meses de janeiro a julho de 2021, utilizou-se o tabulador genérico de domínio público (TABNET) disponível no (DataSUS). Adotou-se como critério de inclusão os casos de sífilis em gestantes no Brasil, durante os anos de 2010 a 2020, notificados no (SINAN). Os critérios de exclusão foram os casos notificados fora do período analisado, em outros anos de notificação, e que não constavam no (SINAN). Para a análise dos casos considerou-se as variáveis: número de casos, detecção, ano, período trimestral, idade gestacional, faixa etária, cor ou raça, classificação clínica, realização de pré-natal. Os dados foram organizados e tabulados utilizando-se o programa Microsoft Excel 2010 e, posteriormente, submetidos à análise estatística descritiva.

Efetuou-se a busca de evidências científicas, através da Plataforma da Biblioteca Virtual em Saúde - BVS, utilizando as bases de dados Literatura Latino-Americana e do Caribe em Ciências da Saúde (LILACS), Base de Dados de Enfermagem (BDENF), por meio da - Scientific Electronic Library Online (SCIELO). Aderindo-se através dos descritores/palavras chaves: "Cuidados de Enfermagem", "Gestante", "Sífilis", combinados com o operador booleano "AND".

A realização de estudos epidemiológicos é muito importante para que sejam identificadas as causas ou a etiologia das doenças e seus fatores de risco. Assim, é possível desenvolver estratégias preventivas que reduzam ou mesmo eliminem a exposição aos fatores de risco. A finalidade primordial desses estudos é a redução da morbidade e mortalidade por agravos ou doenças (Fontelles, Simões, Farias, \& Fontelles, 2019). 
Na pesquisa observacional o investigador atua meramente como expectador de fenômenos ou fatos, sem, no entanto, realizar qualquer intervenção que possa interferir no curso natural e/ou no desfecho dos mesmos, embora possa, neste meio tempo, realizar medições, análises e outros procedimentos para coleta de dados (Fontelles, Simões, Farias, \& Fontelles, 2019).

A pesquisa descritiva é aquela que visa apenas a observar, registrar e descrever as características de um determinado fenômeno ocorrido em uma amostra ou população, sem, no entanto, analisar o mérito de seu conteúdo. Geralmente, na pesquisa quantitativa do tipo descritiva, o delineamento escolhido pelo pesquisador não permite que os dados possam ser utilizados para testes de hipóteses, embora hipóteses possam ser formuladas a posteriori, uma vez que o objetivo do estudo é apenas descrever o fato em si (Fontelles, Simões, Farias, \& Fontelles, 2019).

A Pesquisa documental é o tipo de pesquisa que tem o levantamento de documentos como base. É uma valiosa técnica de coleta de dados qualitativos. Assemelha-se à pesquisa bibliográfica, a qual utiliza a contribuição fornecida por diversos autores sobre um determinado assunto, enquanto na pesquisa documental, a coleta de informações é realizada em materiais que não receberam qualquer tipo de análise crítica. Neste tipo de pesquisa, os documentos consultados são, geralmente, classificados como fontes primárias e fontes secundárias. No primeiro caso, são as fontes cuja origem remonta à época que se está pesquisando, ainda não analisadas e que, frequentemente, foram produzidas pelas próprias pessoas estudadas, tais como correspondências, diários, textos literários e outros documentos mantidos em órgãos públicos e instituições privadas de qualquer natureza; no segundo, correspondem às fontes cujos trabalhos escritos se baseiam na fonte primária, e tem como característica o fato de não produzir informações originais, mas, apenas, uma análise, ampliação e comparação das informações contidas na fonte original (Fontelles, Simões, Farias, \& Fontelles, 2019).

A pesquisa documental se caracteriza pela pesquisa de materiais que não receberam ainda um tratamento analítico, ou que ainda podem ser reelaborados de acordo com os objetos da pesquisa. Além disso, a etapa documental pode complementar a pesquisa, subsidiando dados encontrados por outras fontes, no sentido de corroborar a confiabilidade dos dados. Dessa forma a pesquisa documental é um procedimento metodológico decisivo para algumas áreas. Em muitas pesquisas, os documentos são a única fonte de informação, assim como este tipo de pesquisa também pode ser utilizado de forma associada ou complementar com outros procedimentos metodológicos (Cechinel, Fontana, Giustina, Pereira, \& 2016).

A etapa de análise dos documentos propõe-se a produzir ou reelaborar conhecimentos e criar novas formas de compreender os fenômenos. É condição necessária que os fatos devem ser mencionados, pois constituem os objetos da pesquisa, mas, por si mesmos, não explicam nada. O investigador deve interpretá-los, sintetizar as informações, determinar tendências e na medida do possível fazer a inferência. Os documentos não existem isoladamente, mas precisam ser situados em uma estrutura teórica para que o seu conteúdo seja entendido (Sá Silva, Almeida, \& Guindani, 2009).

A pesquisa qualitativa é o tipo de pesquisa apropriada para quem busca o entendimento de fenômenos complexos específicos, em profundidade, de natureza social e cultural, mediante descrições, interpretações e comparações, sem considerar os seus aspectos numéricos em termos de regras matemáticas e estatísticas. Diferente da quantitativa, a pesquisa qualitativa é mais participativa, porém menos controlável e, por esta razão, tem sido questionada quanto a sua validade e confiabilidade (Fontelles, Simões, Farias, \& Fontelles, 2019).

A pesquisa quantitativa é aquela que trabalha com variáveis expressas sob a forma de dados numéricos e emprega rígidos recursos e técnicas estatísticas para classificá-los e analisá-los, tais como a porcentagem, a média, o desvio padrão, o coeficiente de correlação e as regressões, entre outros. Em razão de sua maior precisão e confiabilidade, os estudos quantitativos são mais indicados para o planejamento de ações coletivas, pois seus resultados são passíveis de generalização, principalmente quando as amostras pesquisadas representam, com fidelidade, a população de onde foram retiradas (Fontelles, Simões, Farias, \& Fontelles, 2019). 
O presente estudo assegura os aspectos éticos, garantindo a autoria dos artigos pesquisados, utilizando para as citações e referências dos autores as normas APA. Os preceitos éticos estabelecidos no que se refere a zelar pela legitimidade das informações, privacidade e sigilo das informações, quando necessárias, tornando os resultados desta pesquisa públicos. Os pesquisadores buscaram a legitimidade e fidelidade nas citações dos autores seja nas citações diretas ou parafraseadas no estudo.

\section{Resultados e Discussão}

Diante dos resultados obtidos no estudo por meio da estratégia de busca, os autores delinearam variáveis para melhor descrever as evidências encontradas na pesquisa. As variáveis propostas são: número de casos, detecção, ano, período trimestral, idade gestacional, faixa etária, cor ou raça, classificação clínica, e realização do pré-natal.

Gráfico 1. Casos e taxa de detecção (por 100.000 habitantes) de sífilis adquirida por ano de diagnóstico. Brasil, 2010-2020.

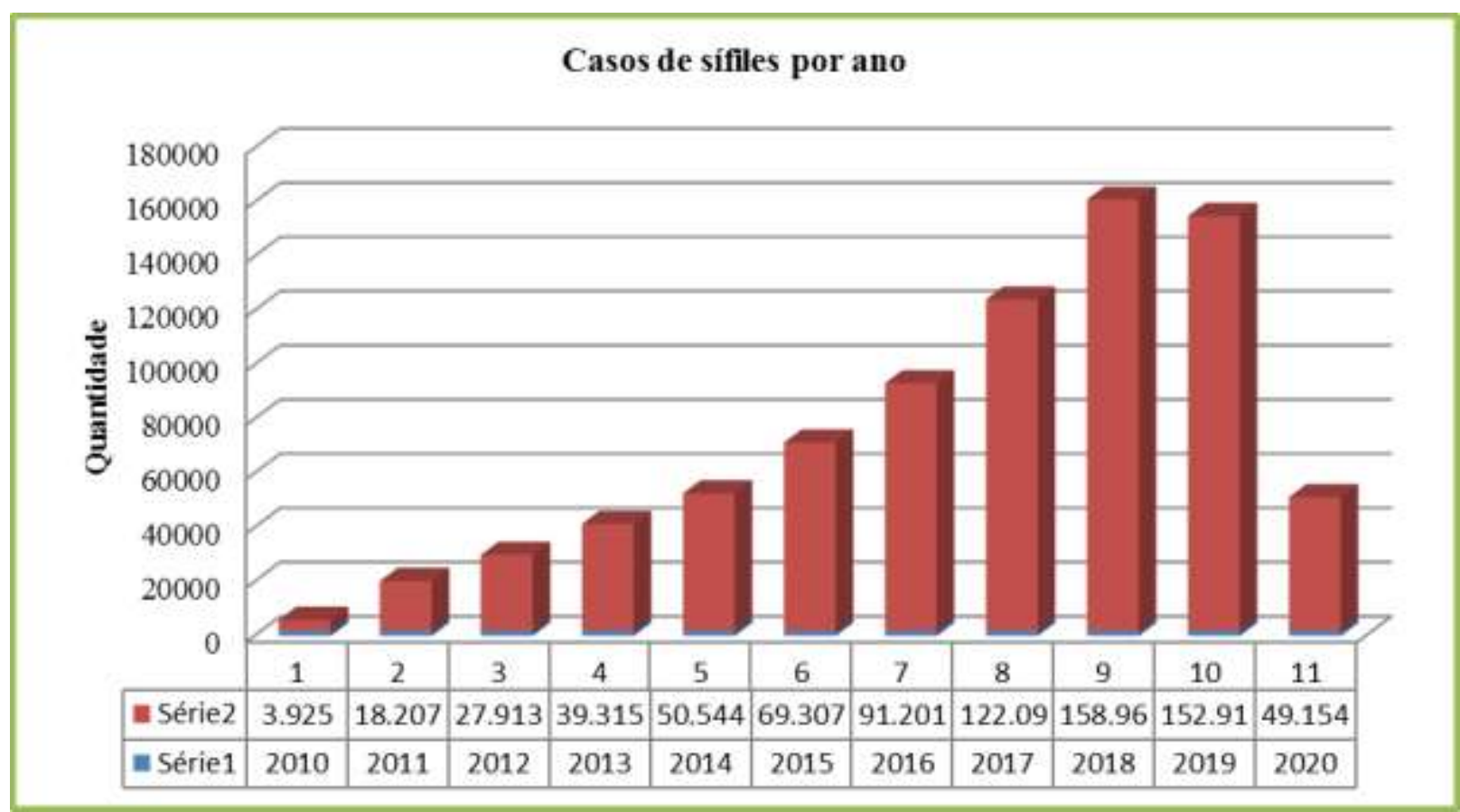

Notas: (1) Dados até 30/06/2020; (2) Dados preliminares para os últimos 5 anos. Fonte: MS/SVS/Departamento de Doenças de Condições Crônicas e Infecções Sexualmente Transmissíveis. Fonte: Autores.

Ao analisar o Gráfico 1, observa-se o número de casos e a taxa de detecção (por 100.000 habitantes), dos casos de sífilis do ano de 2010 a 2020, adquiridas por ano de diagnóstico. Nota-se que no ano de 2010 ocorreu (3925) casos, 2011(18207), 2012 (27913), 2013 (39315), 2014 (50544), 2015 (69307), 2016 (91201), 2017 (12209), 2018 (15896), 2019 (15291), 2020 (49154). 
Research, Society and Development, v. 10, n. 9, e32610917932, 2021

(CC BY 4.0) | ISSN 2525-3409 | DOI: http://dx.doi.org/10.33448/rsd-v10i9.17932

Gráfico 2. Distribuição das taxas de detecção por ano.

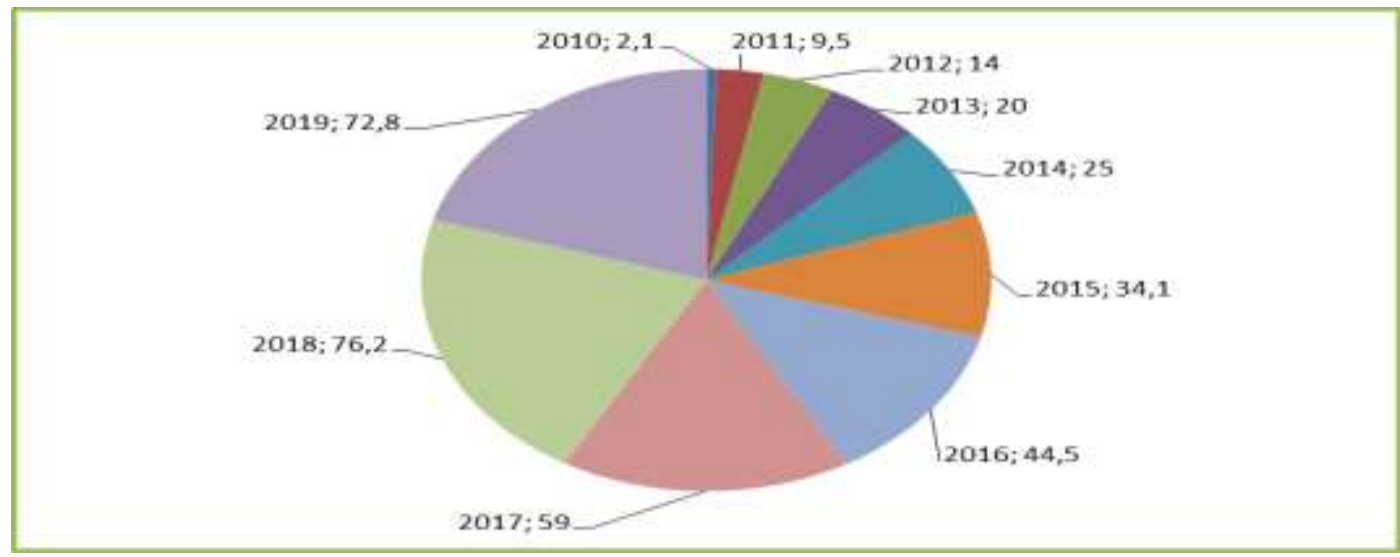

(1) Dados até 30/06/2020; (2) Dados preliminares para os últimos 5 anos. Fonte: MS/SVS/Departamento de Doenças de Condições Crônicas e Infecções Sexualmente Transmissíveis. Notas. Fonte: Autores.

De acordo com o Gráfico 2, percebe-se as taxas de detecção de sífilis por ano, identifica-se que, em 2010 houve (2,1\%), 2011 (9,5\%), 2012 (14\%), 2013 (20\%), 2014 (25\%), 2015 (34,1), 2016 (44,5\%), 2017 (59\%), 2018 (76,2\%), $2019(72,8 \%), 2020(-)$.

Quadro 1. Casos de gestantes com sífiles segundo idade gestacional por ano de diagnóstico. Brasil, 2010 - 2020.

\begin{tabular}{|c|c|c|c|c|c|c|c|c|c|c|c|c|}
\hline Período & $\begin{array}{c}\text { Idade } \\
\text { Gestacional }\end{array}$ & $\mathbf{2 0 1 0}$ & $\mathbf{2 0 1 1}$ & $\mathbf{2 0 1 2}$ & $\mathbf{2 0 1 3}$ & $\mathbf{2 0 1 4}$ & $\mathbf{2 0 1 5}$ & $\mathbf{2 0 1 6}$ & $\mathbf{2 0 1 7}$ & $\mathbf{2 0 1 8}$ & $\mathbf{2 0 1 9}$ & $\mathbf{2 0 2 0}$ \\
\hline $1^{0}$ Trimestre & 130.014 & 2.186 & 3.170 & 3.810 & 5.351 & 7.692 & 10.562 & 14.210 & 19.803 & 24.627 & 23.642 & 10.378 \\
\hline $2^{0}$ Trimestre & 105.732 & 3.299 & 4.318 & 5.097 & 6.645 & 8.164 & 9.764 & 11.003 & 13.896 & 15.863 & 14.788 & 5.686 \\
\hline $3^{0}$ Trimestre & 118.241 & 3.821 & 5.153 & 6.188 & 7.368 & 8.860 & 10.468 & 10.754 & 13.397 & 18.740 & 18.553 & 6.779 \\
\hline IG Ignorada & 24.319 & 764 & 1.107 & 1.337 & 1.541 & 1.900 & 1.977 & 2.238 & 2.555 & 3.663 & 3.972 & 1.292 \\
\hline Ignorado & 461 & - & - & - & - & - & 3 & 54 & 54 \\
\hline
\end{tabular}

(1) Dados até 30/06/2020; (2) Dados preliminares para os últimos 5 anos. Fonte: MS/SVS/Departamento de Doenças de Condições Crônicas e Infecções Sexualmente Transmissíveis. Notas. Fonte: Autores.

Ao analisar o Quadro 1, observa-se a distribuição dos casos de gestantes com sífilis de acordo com a idade gestacional por ano de diagnóstico no Brasil durante os anos de 2010 a 2020. 
Research, Society and Development, v. 10, n. 9, e32610917932, 2021

(CC BY 4.0) | ISSN 2525-3409 | DOI: http://dx.doi.org/10.33448/rsd-v10i9.17932

Tabela 1. Distribuição percentual de casos de gestantes com sífilis segundo idade gestacional por ano de diagnóstico. Brasil, 2010-2020.

\begin{tabular}{|c|c|c|c|c|c|c|c|c|c|c|c|c|c|}
\hline & Idade Gestacional & $2007-2009$ & 2010 & 2011 & 2012 & 2013 & 2014 & 2015 & 2016 & 2017 & 2018 & 2019 & 2020 \\
\hline $1^{\circ}$ Trimestre & 34,3 & 20,9 & 21,7 & 23,1 & 23,2 & 25,6 & 28,9 & 32,2 & 37,1 & 39,8 & 39,1 & 38,7 & 42,9 \\
\hline $2^{\circ}$ Trimestre & 27,9 & 32,9 & 32,8 & 31,4 & 31,0 & 31,8 & 30,7 & 29,8 & 28,8 & 27,9 & 25,2 & 24,2 & 23,5 \\
\hline $3^{0}$ Trimestre & 31,2 & 37,2 & 37,9 & 37,5 & 37,7 & 35,2 & 33,3 & 31,9 & 28,1 & 26,9 & 29,8 & 30,4 & 28,0 \\
\hline Idade gestacional ignorada & 6,4 & 9,0 & 7,6 & 8,1 & 8,1 & 7,4 & 7,1 & 6,0 & 5,8 & 5,1 & 5,8 & 6,5 & 5,3 \\
\hline Ignorado & 0,1 & 0,0 & . & - & - & . & . & 0,0 & 0,1 & 0,2 & 0,1 & 0,3 & 0,2 \\
\hline
\end{tabular}

(1) Dados até 30/06/2020; (2) Dados preliminares para os últimos 5 anos. Fonte: MS/SVS/Departamento de Doenças de Condições Crônicas e Infecções Sexualmente Transmissíveis. Notas. Fonte: Autores.

De acordo com a Tabela 1, identifica-se o percentual dos casos de gestantes segundo a idade gestacional por ano de diagnóstico no Brasil durante os anos de 2010 a 2020.

Tabela 2. Distribuição percentual de casos de gestantes com sífilis segundo faixa etária por ano de diagnóstico. Brasil, 20102020 .

\begin{tabular}{|c|c|c|c|c|c|c|c|c|c|c|c|c|c|}
\hline Faixa Etária & Total & $2005-2009$ & 2010 & 2011 & 2012 & 2013 & 2014 & 2015 & 2016 & 2017 & 2018 & 2019 & 2020 \\
\hline 10 a 14 anos & 1,3 & 1,3 & 1,7 & 1,5 & 1,6 & 1,6 & 1,4 & 1,4 & 1,4 & 1,2 & 1,1 & 1,0 & 1,0 \\
\hline 15 a 19 anos & 24,5 & 18,6 & 21,0 & 22,5 & 24,2 & 25,6 & 26,3 & 26,0 & 26,0 & 26,0 & 24,9 & 24,0 & 23,8 \\
\hline 20 a 29 anos & 53,0 & 53,7 & 52,0 & 50,8 & 50,4 & 50,5 & 50,4 & 51,4 & 52,5 & 52,8 & 53,8 & 55,7 & 55,6 \\
\hline 30 a 39 anos & 19,1 & 23,2 & 22,6 & 22,2 & 21,4 & 20,4 & 19,9 & 19,1 & 18,3 & 18,0 & 18,2 & 17,4 & 17,6 \\
\hline 40 anos ou mais & 2,1 & 3,1 & 2,7 & 2,9 & 2,4 & 2,0 & 2,1 & 2,0 & 1,9 & 2,0 & 1,9 & 1,9 & 1,9 \\
\hline Ignorado & 0,0 & 0,0 & - & - & - & - & . & - & 0,0 & 0,0 & 0,0 & . & . \\
\hline
\end{tabular}

(1) Dados até 30/06/2020; (2) Dados preliminares para os últimos 5 anos. Fonte: MS/SVS/Departamento de Doenças de Condições Crônicas e Infecções Sexualmente Transmissíveis. Notas. Fonte: Autores.

Segundo a Tabela 2, pode-se analisar a distribuição do percentual de casos de gestantes com sífilis de acordo com a faixa etária por ano de diagnóstico no Brasil, nos anos de 2010 a 2020. 
Tabela 3. Casos de gestantes com sífilis segundo cor ou raça por ano de diagnóstico. Brasil, 2010-2020.

\begin{tabular}{|c|c|c|c|c|c|c|c|c|c|c|c|c|c|}
\hline Raça ou Cor & Total & $2005-2009$ & 2010 & 2011 & 2012 & 2013 & 2014 & 2015 & 2016 & 2017 & 2018 & 2019 & 2020 \\
\hline Branca & 113.296 & 7.888 & 2.967 & 3.923 & 4.766 & 6.241 & 8.003 & 10.119 & 11.802 & 15.289 & 18.116 & 17.507 & 6.675 \\
\hline Preta & 46.991 & 3.040 & 1.219 & 1.786 & 2.098 & 2.484 & 3.362 & 3.975 & 4.788 & 6.321 & 7.705 & 7.258 & 2.955 \\
\hline Amarela & 3.505 & 332 & 67 & 108 & 134 & 186 & 203 & 262 & 323 & 458 & 608 & 582 & 242 \\
\hline Parda & 186.601 & 12.101 & 4.710 & 6.359 & 7.633 & 9.711 & 12.441 & 15.308 & 18.033 & 24.193 & 32.024 & 31.279 & 12.809 \\
\hline Indígena & 2.797 & 611 & 149 & 148 & 133 & 130 & 152 & 225 & 227 & 266 & 331 & 310 & 115 \\
\hline Ignorada & 31.220 & 3.299 & 958 & 1.424 & 1.668 & 2.153 & 2.455 & 2.886 & 3.107 & 3.288 & 4.398 & 4.191 & 1.393 \\
\hline
\end{tabular}

(1) Dados até 30/06/2020; (2) Dados preliminares para os últimos 5 anos. Fonte: MS/SVS/Departamento de Doenças de Condições Crônicas e Infecções Sexualmente Transmissíveis. Notas. Fonte: Autores.

Ao analisar a Tabela 3, nota-se a distribuição dos casos de gestantes com sífilis segundo cor ou raça por ano de diagnóstico no Brasil durante os anos de 2010-2020.

Tabela 4. Distribuição percentual de casos de gestantes com sífilis segundo cor ou raça por ano de diagnóstico. Brasil, 20102020 .

\begin{tabular}{|c|c|c|c|c|c|c|c|c|c|c|c|c|c|}
\hline Raça ou Cor & Total & $2005-2009$ & 2010 & 2011 & 2012 & 2013 & 2014 & 2015 & 2016 & 2017 & 2018 & 2019 & 2020 \\
\hline Branca & 29,5 & 28,9 & 29,5 & 28,5 & 29,0 & 29,9 & 30,1 & 30,9 & 30,8 & 30,7 & 28,7 & 28,6 & 27,6 \\
\hline Preta & 12,2 & 11,1 & 12,1 & 13,0 & 12,8 & 11,9 & 12,6 & 12,1 & 12,5 & 12,7 & 12,2 & 11,9 & 12,2 \\
\hline Amarela & 0,9 & 1,2 & 0,7 & 0,8 & 0,8 & 0,9 & 0,8 & 0,8 & 0,8 & 0,9 & 1,0 & 1,0 & 1,0 \\
\hline Parda & 48,5 & 44,4 & 46,8 & 46,3 & 46,5 & 46,5 & 46,7 & 46,7 & 47,1 & 48,6 & 50,7 & 51,2 & 53,0 \\
\hline Indígena & 0,7 & 2,2 & 1,5 & 1,1 & 0,8 & 0,6 & 0,6 & 0,7 & 0,6 & 0,5 & 0,5 & 0,5 & 0,5 \\
\hline Ignorada & 8,1 & 12,1 & 9,5 & 10,4 & 10,2 & 10,3 & 9,2 & 8,8 & 8,1 & 6,6 & 7,0 & 6,9 & 5,8 \\
\hline
\end{tabular}

(1) Dados até 30/06/2020; (2) Dados preliminares para os últimos 5 anos. Fonte: MS/SVS/Departamento de Doenças de Condições Crônicas e Infecções Sexualmente Transmissíveis. Notas. Fonte: Autores.

De acordo com a Tabela 4, pode-se analisar a distribuição do percentual de casos de gestantes com sífilis segundo cor ou raça por ano de diagnóstico no Brasil durante os anos de 2010-2020. 
Tabela 5. Casos de gestantes com sífilis segundo classificação clínica por ano de diagnóstico. Brasil, 2010-2020.

\begin{tabular}{|c|c|c|c|c|c|c|c|c|c|c|c|c|c|}
\hline Classificação Clínica & Total & $2007-2009$ & 2010 & 2011 & 2012 & 2013 & 2014 & 2015 & 2016 & 2017 & 2018 & 2019 & 2020 \\
\hline Sífilis Primária & 111.836 & 8.665 & 3.784 & 4.852 & 5.684 & 6.795 & 8.507 & 10.099 & 11.151 & 14.093 & 16.725 & 15.315 & 6.166 \\
\hline Sífilis Terciária & 36.231 & 1.361 & 809 & 1.102 & 1.335 & 2.197 & 3.003 & 3.500 & 4.110 & 5.389 & 6.109 & 5.108 & 2.208 \\
\hline Ignorado & 100.974 & 6.861 & 3.225 & 4.544 & 5.106 & 6.192 & 7.443 & 9.183 & 10.239 & 12.527 & 15.551 & 14.668 & 5.435 \\
\hline
\end{tabular}

(1) Dados até 30/06/2020; (2) Dados preliminares para os últimos 5 anos. Fonte: MS/SVS/Departamento de Doenças de Condições Crônicas e Infecções Sexualmente Transmissíveis. Notas. Fonte: Autores.

Ao analisar a Tabela 5, pode-se identificar o número dos casos de gestantes com sífilis segundo classificação clínica por ano de diagnóstico no Brasil durante os anos de 2010-2020.

Tabela 6. Distribuição percentual de casos de gestantes com sífilis segundo classificação clínica por ano de diagnóstico. Brasil, 2010-2020.

\begin{tabular}{|c|c|c|c|c|c|c|c|c|c|c|c|c|c|}
\hline Classificação Clínica & Total & $2007-2009$ & 2010 & 2011 & 2012 & 2013 & 2014 & 2015 & 2016 & 2017 & 2018 & 2019 & 2020 \\
\hline Sífilis Primária & 29,5 & 39,5 & 37,6 & 35,3 & 34,6 & 32,5 & 32,0 & 30,8 & 29,1 & 28,3 & 26,5 & 25,1 & 25,5 \\
\hline Sífilis Secundária & 5,7 & 8,4 & 7,6 & 6,6 & 6,7 & 6,3 & 6,2 & 5,8 & 5,6 & 5,3 & 5,0 & 4,8 & 4,3 \\
\hline Sifilis Terciária & 9,6 & 6,2 & 8,0 & 8,0 & 8,1 & 10,5 & 11,3 & 10,7 & 10,7 & 10,8 & 9,7 & 8,4 & 9,1 \\
\hline Ignorado & 26,6 & 31,3 & 32,0 & 33,1 & 31,1 & 29,6 & 28,0 & 28,0 & 26,7 & 25,1 & 24,6 & 24,0 & 22,5 \\
\hline
\end{tabular}

(1) Dados até 30/06/2020; (2) Dados preliminares para os últimos 5 anos. Fonte: MS/SVS/Departamento de Doenças de Condições Crônicas e Infecções Sexualmente Transmissíveis. Notas. Fonte: Autores.

De acordo com a Tabela 6, nota-se a distribuição percentual dos casos de gestantes com sífilis segundo classificação clínica por ano de diagnóstico no Brasil durante os anos de 2010-2020.

Tabela 7. Casos de sífilis congênita segundo informação sobre realização de pré-natal da mãe por ano de diagnóstico. Brasil, 2010-2020.

\begin{tabular}{|c|c|c|c|c|c|c|c|c|c|c|c|c|c|}
\hline Realizaçāo de prénatal & Total & $2007-2009$ & 2010 & 2011 & 2012 & 2013 & 2014 & 2015 & 2016 & 2017 & 2018 & 2019 & 2020 \\
\hline $\mathrm{Sin}$ & 186.740 & 42.584 & 5,056 & 6.094 & 8.557 & 10.452 & 12606 & 15,179 & 17323 & 20.455 & 21683 & 20.163 & 7276 \\
\hline Kâ० & 36.948 & 9.374 & 1398 & 1.842 & 2411 & 2597 & 2745 & 2931 & $28 B 4$ & 3.296 & 3.554 & 2.896 & 1060 \\
\hline $\lg n a r a d a$ & 14,028 & 3.409 & 507 & 280 & 710 & 929 & 942 & 1302 & 1.123 & 1295 & 1294 & 2.194 & 632 \\
\hline
\end{tabular}

(1) Dados até 30/06/2020; (2) Dados preliminares para os últimos 5 anos. Fonte: MS/SVS/Departamento de Doenças de Condições Crônicas e Infecções Sexualmente Transmissíveis. Notas. Fonte: Autores.

Segundo a Tabela 7, pode-se analisar a distribuição do número dos casos de sífilis congênita segundo informação sobre realização de pré-natal da mãe por ano de diagnóstico no Brasil durante os anos de 2010-2020. 
Tabela 8. Distribuição percentual de casos de sífilis congênita segundo informação sobre realização de pré-natal da mãe por ano de diagnóstico. Brasil, 2010-2020.

\begin{tabular}{|c|c|c|c|c|c|c|c|c|c|c|c|c|c|}
\hline Realizaçao de pré-natal & Total & $2007-2009$ & 2010 & 2011 & 2012 & 2913 & 2014 & 2015 & 2016 & 2017 & 2018 & 2019 & 2020 \\
\hline Sm & 78,6 & 3428 & 73,1 & 73,4 & 73,3 & 74,8 & 77,5 & 78,5 & 812 & 81,7 & 81,7 & 83,1 & 81,1 \\
\hline N50 & 15,5 & 79,2 & 19,6 & 19,4 & 20,6 & 18,5 & 26,8 & 149 & 13,5 & 131 & 13,4 & 11,9 & 118 \\
\hline Ignorado & 5,9 & 28,8 & 7,3 & 7,3 & 6,1 & 6,6 & 5,8 & 6,6 & 5,3 & 52 & 49 & 49 & 7,0 \\
\hline
\end{tabular}

(1) Dados até 30/06/2020; (2) Dados preliminares para os últimos 5 anos. Fonte: MS/SVS/Departamento de Doenças de Condições Crônicas e Infecções Sexualmente Transmissíveis. Notas. Fonte: Autores.

Ao analisar a Tabela 8, pode-se notar a distribuição percentual dos casos de sífilis congênita segundo informação sobre realização de pré-natal da mãe por ano de diagnóstico no Brasil durante os anos de 2010-2020.

O tratamento e a detecção da sífilis na gestação são representativos de grande importância para a saúde pública, sendo essencial para a prevenção e o controle desta patologia. Os profissionais enfermeiros possuem papel fundamental quanto à aplicação de estratégias que auxiliem na prevenção e no diagnóstico da sífilis congênita, assim como, reduzir os riscos de mortalidade e morbidade materna e fetal. É necessário e de grande importância que exista uma assistência de enfermagem voltada para gestantes e parceiros com a realização de ações por parte dos profissionais de saúde em especial o enfermeiro, promovendo uma melhor qualidade, com rastreamento da sífilis na consulta pré-natal, atividades ligadas à educação em saúde, controle de casos da doença, realizando sempre a notificação, busca ativa, um tratamento correto dos parceiros sexuais, acompanhamento e monitoramento de exames sorológicos para confirmação de possível cura (Nesi, Graf, \& Moraes, 2020).

De acordo com Gomes, Oliveira, e Brandão (2020), modificação psicológicas e biológicas permeiam o organismo feminino no período da gestação criando vínculos e estabelecendo a comunicação entre o binômio mãe-filho, favorecendo a atuação da enfermagem nesse processo. As infecções gestacionais acarretam inúmeras consequências podendo ocorrer prejuízos para mãe e o filho.

Segundo Nascimento, Nunes, Oliveira, Rita, e Dias (2017), a sífilis gestacional vem ocorrendo com frequência destacando-se alguns fatores que contribuem, estes são: classe média baixa, baixo índice de escolaridade, déficit no acompanhamento pré-natal, sendo este um dos mais importantes para o desfecho de intervir no resultado, adquirida no protocolo da gestante, garantido desta forma uma assistência qualificada.

Estima-se que grande parte das gestações com sífilis não tratada evoluam para perda fetal e que mais de $50 \%$ dos recém-nascidos de mães com sífilis não tratada ou tratada de forma inadequada, não apresentem sinais evidentes da doença logo ao nascimento, retardando o seu diagnóstico e, produzindo consequentemente, complicações para a criança no futuro. A assistência de enfermagem é de extrema importância, visto que, a qualidade da assistência na gestação e parto é um vetor determinante na redução da transmissão vertical da sífilis e de outras doenças infectocontagiosas, ressaltando-se a importância da notificação, pois é através desses dados epidemiológicos que medidas devem ser tomadas para o controle de casos futuros (Gomes, Oliveira, \& Brandão, 2020)

A sífilis é uma patologia multifacetada, com sérias implicações para a mulher grávida e seu concepto. Quando adquirida durante a gravidez, pode levar a abortamento espontâneo, morte fetal e neonatal, prematuridade e danos à saúde do recém-nascido com repercussões psicológicas e sociais. A sífilis gestacional é considerada quando toda gestante com evidência clínica de sífilis e/ou com sorologia não treponêmica reagente, com qualquer titulagem, mesmo na ausência de resultado de teste treponêmico, realizada no pré-natal ou no momento do parto ou curetagem. A transmissão ocorre, na maioria dos casos, 
pela via sexual e se dá pela penetração da bactéria através de microscópicas feridas ou abrasões na mucosa da vagina ou do pênis. Se não tratada, a doença pode evoluir a estágios que comprometem a pele e órgãos internos, como o coração, fígado e sistema nervoso central (Gomes, Oliveira, \& Brandão, 2020)

No entanto, a assistência de enfermagem no pré-natal é de suma importância na primeira consulta, avaliando os parâmetros necessários em busca de alguma alteração, o resulto positivo da sífilis, que pode culminar em morte do RN e má formação congênita. É de suma relevância o tratamento do parceiro e acompanhamento laboratorial do mesmo, pois o contato sexual desprotegido, pode ocasionar reinfecção da gestante, agravando a infecção e aumentando a possibilidade de transmissão vertical. Deste modo, é importante fornecer assistência ao casal, dando-lhe um suporte adequado tanto eficácia no tratamento como no apoio psicossocial, investigando histórico e necessidades a fim de traçar uma assistência de enfermagem sistematizada (Nascimento, Nunes, Oliveira, Rita, \& Dias, 2017).

Em se tratando do controle da sífilis congênita o profissional de enfermagem atua em diversas frentes. As ações educativas desenvolvidas vão desde as palestras para grupos de gestantes, a visitas domiciliares para educação das futuras mães bem como a realização e monitoramento constante e de perto das gestantes através da realização dos testes rápidos (TR) periódicos, bem como a garantia de tratamento para casos positivos para sífilis seguindo os protocolos do Ministério da Saúde. Assim, o profissional de enfermagem tem papel primordial no que se refere à prevenção e ao diagnóstico da sífilis congênita, dentro da Estratégia de Saúde da Família (ESF) é o profissional que realiza o primeiro contato com as gestantes e são os responsáveis pela execução das ações de prevenção individual e coletiva, ações educativas com palestras sobre a sífilis, através da ESF, escolas, reuniões em comissões locais nos bairros e nas visitas domiciliares (Souza et al., 2018).

A forma mais eficiente de prevenção são os cuidados nas relações sexuais. O uso de preservativo e evitar ações de promiscuidade são fundamentais neste processo. Em relação às gestantes, a realização de testes no período de pré-natal é primordial em prol de diagnóstico precoce e assim permitir o tratamento das gestantes positivas para a sífilis, evitando-se assim a transmissão vertical. O MS preconiza que as pessoas sexualmente ativas devem realizar o teste para sífilis, em especial, as gestantes alertando que a sífilis pode levar ao aborto, má formação fetal do feto/ou morte ao nascer. Logo o exame deve ser realizado na primeira consulta de pré-natal, no terceiro trimestre da gestação e no momento do parto, independente dos testes anteriores (Souza et al., 2018).

Dessa forma, quando uma paciente gestante tem diagnóstico positivo para sífilis é necessário que a mesma seja assistida por um profissional com conhecimento e habilidades específicas no que se refere ao manejo, cuidado e tratamento desta patologia. Por sua vez o profissional de enfermagem é habilitado e designado legalmente para realização do pré-natal das gestantes de baixo risco. O enfermeiro é o profissional imprescindível no que se refere à prevenção da sífilis, visto que o mesmo tem acesso à gestante através do Agente Comunitário de Saúde (ACS) cujas visitas domiciliares permitem levar ao enfermeiro informações a respeito dos cuidados domiciliares referentes ao tratamento das pacientes cujos VDRL foram positivos na consulta do pré-natal. A ação educativa do profissional de enfermagem é estritamente relevante no que se refere à prevenção e cuidados frente à sífilis congênita. Pode-se sintetizar como ações específicas ao profissional de enfermagem na prevenção da sífilis congênita, a realização das consultas de enfermagem de pré-natal e o aconselhamento e seguimento das pacientes gestantes cujo teste de VDRL foi positivo. Além disso, cabe ao profissional de enfermagem atuar a partir da orientação educacional visando à prevenção de casos novos de sífilis, através da prática de orientações e informações adequadas a todas as mulheres e seus parceiros (Souza et al., 2018).

Nesse aspecto, percebeu-se a necessidade de politicas públicas que promovam a sensibilização e capacitação da equipe de saúde voltada para assistência pré-natal, visando capacita-los quanto à notificação e manejo clínico da sífilis no curso da gestação. Em relação à captação dos parceiros para o tratamento, verificou-se a necessidade de promover estratégias mais eficazes para o tratamento, como por exemplo, sensibilização, aconselhamento e preparação da enfermeira para atender ás 
vulnerabilidades deste grupo (Suto, Silva, Almeida, Costa, \& Evangelista, 2016).

Assim é preciso que o enfermeiro busque ampliar seu conhecimento acerca da doença, através de cursos e atualizações, pois este profissional tem um papel decisivo na prevenção, diagnóstico, tratamento e quebra da cadeia de transmissão da sífilis. O enfermeiro deve também implementar estratégias eficazes de abordagem ao parceiro, educando e estimulando a criação de vínculo, buscando o resgate das relações humanas e possibilitar espaços para escuta e discussões sobre a fragilidade dos relacionamentos humanos na sociedade e a repercussão do mesmo na esfera social e individual (Solino et al., 2020).

As estratégias do enfermeiro para o combate à sífilis não se resumem apenas em curar o doente, porém em realizar uma consulta de enfermagem de qualidade ações que vão além do âmbito de espaço e acolhimento. Em relação à consulta de enfermagem de qualidade e importante que o profissional sempre realize um atendimento único, humanizado e personalizado para o usuário, levando em consideração o meio social em que o usuário se encontra para que não possa ter resistência ao tratamento e por fim quebrar a cadeia de transmissão da sífilis. As ações de enfermagem são importantes para o rastreamento e controle de casos, que existem em campanhas envolvendo a saúde da comunidade e propagando a educação em saúde buscando a adesão dos usuários ao tratamento medicamentos para a sífilis (Silva Júnior, Lima, \& Aramaio, 2021).

O combate à sífilis deve ser de ações conjuntas dos gestores e profissionais de saúde. Os gestores oferecendo subsídios para o diagnóstico e tratamento de forma adequada e os profissionais de saúde buscar capacitação para prestar uma assistência de qualidade para os usuários. O enfermeiro como responsável da assistência de enfermagem de qualidade, tem como objetivo formular estratégias com a equipe da atenção primária para realizar a buscar ativa dos parceiros que se recusam a acompanhar a gestante ao pré-natal, oferecendo-os recursos necessários para a realização do teste e o tratamento. Levando em consideração que uma vez que a cadeia de transmissão é quebrada os indicadores de contaminação na coletividade diminuem, podendo mostrar o resultado dos esforços dos profissionais em frente ao combate da sífilis (Silva Júnior, Lima, \& Aramaio, 2021).

A participação do enfermeiro é fundamental para fortalecimento da atenção pré-natal, haja vista que esta consulta se dará com identificação dos fatores de riscos gestacionais a fim de que sejam diminuídas implicações na saúde das gestantes, em especial aquelas com sífilis. Deste modo, a atuação do enfermeiro proporciona extensão na cobertura e melhoria da qualidade na atenção pré-natal, o qual deve estar ciente de que a atenção qualificada e humanizada se dá por meio da incorporação de condutas acolhedoras, sem intervenções desnecessárias, fácil acesso a serviços de saúde de qualidade com ações que integrem todos os níveis da atenção, como promoção, prevenção e assistência à saúde da gestante e recém-nascido desde o atendimento ambulatorial básico ao hospitalar para alto risco (Nunes et al., 2017).

O acompanhamento é imprescindível à gestante com sífilis. Nesse momento, o enfermeiro que atende estas mulheres interroga sobre seu estado, avalia a efetividade e adesão ao tratamento, como também do parceiro. A referida categoria apresenta considerações sobre as ações dos enfermeiros perante a gestante com sífilis durante o pré-natal, configurando-se como principal expoente de seus discursos. Dessa forma, a captação e as orientações às gestantes e parceiro por parte do enfermeiro têm contribuição decisiva para ações eficientes no combate à sífilis, de maneira a ser implementada relação harmoniosa e ética sustentada no compromisso seguro da usuária com resolubilidade de seus problemas. Assim, o enfermeiro é importante no controle da sífilis gestacional informando sobre o tratamento de forma correta; uso de preservativos nas relações sexuais; promover educação em saúde; prática de exames; e captação dos parceiros (Nunes et al., 2017).

Segundo Pereira, Santos \& Gomes (2020), a sífilis gestacional tem importância significativa para a saúde pública, devido ao seu impacto durante a gravidez. Ela pode afetar o desenvolvimento do feto e do RN, aumentando a suscetibilidade ao aborto, parto prematuro, malformações esqueléticas, meningite e pneumonia. Uma das medidas de prevenção cabíveis ao enfermeiro é a realização do teste rápido para sífilis em cada trimestre gestacional. Eles possibilitam a tomada de medidas de 
tratamento há tempo, evitando assim, a SC, prevenindo a Transmissão-Vertical.

\section{Conclusão}

Evidenciou-se no estudo, que a sífilis é uma doença grave, de notificação compulsória em casos de infecção de gestantes e por consequência as crianças. O tratamento é de fácil acesso, baixo custo e eficaz, porém a adesão a este muitas vezes não se concretiza. Nos casos de gestantes infectadas com o Treponema pallidum, que não realiza tratamento ou em quem o tratamento é feito inadequadamente, os agravos na saúde da mulher variam de acordo com o tempo e evolução da doença, sendo que uma das consequências é a ocorrência do aborto espontâneo durante a gestação. O enfermeiro possui um importante papel no controle da sífilis em gestante e o uso de algumas estratégias específicas o auxilia a atingir esse objetivo.

Nesse sentido, o diagnóstico da sífilis durante a gestação merece atenção por parte dos profissionais da saúde, tendo em vista que diversas complicações podem ser evitadas. É necessária que haja uma maior valorização desta doença, sendo de grande importância a conscientização dos profissionais de saúde que atendem as gestantes no pré-natal. Dessa forma, a educação em saúde é importante no desfecho de uma gestação, sendo o profissional enfermeiro, capaz de auxiliar a gestante a viver de forma mais saudável e com todos os cuidados necessários nesse período da sua vida. Portanto, é necessário realizar ações de promoção à saúde na gestação, dando enfoque ao autocuidado e prevenção, pois reduzem os gastos com assistência de maior complexidade e também diminuem as complicações obstétricas e neonatais que podem ser causadas pela sífilis.

Torna-se importante a realização de estudos futuros com a finalidade de ampliar a produção científica a respeito dessa temática tão relevante, sendo de suma importância, no sentido de aprofundar discussões que possam contribuir para a promoção da saúde e na melhoria da qualidade de vida dessas pacientes.

\section{Referências}

Castro Felicio, F., Alves, V. H., Pereira, A. V., Rodrigues, D. P., de Paula, E., \& Almeida, V. L. M. (2019). Percepção da fragilidade da Sistematização da Assistência em Enfermagem: obstáculo no controle da sífilis na gestação. Revista Renome, 8(2), 40-47.

Fontelles, M. J., Simões, M. G., Farias, S. H., \& Fontelles, R. G. S. (2009). Metodologia da pesquisa científica: diretrizes para a elaboração de um protocolo de pesquisa. Revista paraense de medicina, 23(3), 1-8.

Gomes, L. P. D. S., \& Oliveira, S. S. A. D. (2020). A qualidade da assistência da enfermagem no acompanhamento da gestante com sífilis reagente da gravidez até o parto.

Leite, I. A., de melo Leão, M. C., de Oliveira, J. M., \& de França, A. M. B. (2016). Assistência de enfermagem na sífilis na gravidez. Caderno de GraduaçãoCiências Biológicas e da Saúde-UNIT-ALAGOAS, 3(3), 165.

Nascimento, J. S., Nunes, A. J. M., Oliveira, C. P. S., Santa Rita, K. B., \& de Jesus Dias, J. (2017). Sistematização da Assistência de Enfermagem para a Gestante com Sífilis. In Congresso Internacional de Enfermagem.

Nesi, A. N., Graf, M. M. T., \& Moraes, N. A. Assistência do enfermeiro a gestantes com sífilis.

Nunes, J. T., Marinho, A. C. V., Davim, R. M. B., Silva, G. G. D. O., Felix, R. S., \& Martino, M. M. F. D. (2017). Sífilis na gestação: perspectivas e condutas do enfermeiro. Rev. enferm. UFPE on line, 4875-4884.

Oliveira, J. A. C., dos Reis Nunes, C., \& Andrade, C. C. F. (2017). Assistência de enfermagem no pré-natal em relação à sífilis congênita. Múltiplos Acessos, 2(2).

Pereira, A. S., Shitsuka, D. M., Parreira, F. J., \& Shitsuka, R. (2018). Metodologia da pesquisa científica.

Sá-Silva, J. R., Almeida, C. D. D., \& Guindani, J. F. (2009). Pesquisa documental: pistas teóricas e metodológicas. Revista brasileira de história \& ciências sociais, 1(1), 1-15.

Silva Júnior, E. D. A., Lima, R. S., \& de Oliveira Aramaio, C. M. S. (2021). Desafios da enfermagem na assistência da sífilis gestacional na atenção primária de saúde: revisão integrativa. Revista Eletrônica Acervo Enfermagem, 11, e7392-e7392.

Solino, M. D. S. S., Santos, N. D. S. S., dos Santos Almeida, M. C., Santos, L. F., das Graças Gonçalves, J., Pereira, R. S. F., \& de Assunção, M. A. (2020). Desafios do enfermeiro na assistência de enfermagem aos usuários com diagnóstico de sífilis. Brazilian Journal of Health Review, 3(5), 13917-13930.

Souza, L. A., Oliveira, I. S. B., Lenza, N. D. F. B., Rosa, W. D. A. G., Carvalho, V. V., \& Zeferino, M. G. M. (2018). Ações de enfermagem para prevenção da sífilis congênita. Revista de Iniciação Científica da Libertas, 8(1), 108.

Suto, C. S. S., Silva, D. L. D., Almeida, E. D. S. D., Costa, L. E. L., \& Evangelista, T. J. (2016). Assistência pré-natal a gestante com diagnóstico de sífilis. Rev. enferm. atenção saúde, 18-33. 